\title{
INFRARED ABSORPTION ON SHALLOW DONORS IN $\mathrm{CdF}_{2}{ }^{*}$
}

\author{
S. Bednarek AND J. ADAmowski \\ Department of Solid State Physics, Academy of Mining and Metallurgy, \\ al. Mickiewicza 30, 30-059 Kraków, Poland
}

(Received August 8, 1990)

\begin{abstract}
A theoretical description of shallow donor absorption spectra in $\mathrm{CdF}_{2}$ in the infrared region is given. The properties of the stable ( $Y$ ) and bistable (In) donors are analyzed and quantatively described. It is shown that the bistability of donors and the shape of the infrared absorption band have the same origin, which is the combined effect of electron-LO-phonon coupling, conduction band non-parabolicity and short-range central-cell potential.
\end{abstract}

PACS numbers: 71.55.-i.

The absorption spectra due to donors in $\mathrm{CdF}_{2}$ possess many characteristic features [1]. One of them results from the bistability of donors and has been discussed in the previous paper [2]. Another one relies on the large difference in the absorption band shapes between the stable (yttrium) and bistable (indium) donors. For these donors the transition energies between $1 s$ and $n p$ hydrogen-like states differ by $5 \mathrm{meV}$, i.e. merely $5 \%$ of the $1 \mathrm{~s}$ ionisation energy. On the other hand, the absorption band resulting from the transitions between the discrete donor states and conduction band states is much broader for the bistable donor (In) and has a maximum shifted towards high energy by about $75 \mathrm{meV}$ with respect to the stable donor (Y). This effect is qualitatively clear and results from the following argumentation. The chemical shift of the hydrogen-like spectrum results from the short-range potential of the impurity atom core, which also changes a degree of localization of the $1 s$ shallow donor state. This, in turn, leads to the shift of absorption band. However, the observed [1] difference between the absorption bands of both donors is surprisingly large. The shift of the absorption band maximum, calculated in the hydrogen-like model of the donor, is $7 \mathrm{meV}$, while the experimentally observed value is of the order of magnitude larger. The present paper provides an explanation of this effect.

*This work was supported by CPBP 01.04. 
The calculations are performed in the effective mass approximation taking into account the interaction of the electron and donor center with LO phonons, the non-parabolicity of the conduction band and the central-cell corrections. The coupling with LO phonons is assumed in the form of the Fröhlich Hamiltonian taking on the following values of the material parameters: static dielectric constant $\varepsilon_{0}=7.78$, optical dielectric constant $\varepsilon_{\infty}=2.4$, and LO-phonon energy $\hbar \omega=50 \mathrm{meV}$. The values of the remaining material parameters, i.e. conduction band mass and short-range potential of the impurity, have been chosen from the discrete part of the donor spectrum assuming the most natural identification of the first two peaks at $65(70) \mathrm{meV}$ and $75.6(80.5) \mathrm{meV}$ for $\mathrm{Y}$ (In) as $1 s-2 p$ and $1 s-3 p$ transitions, respectively. The $p$-states are not influenced by the short-range potential and form the hydrogen-like spectrum with energies $E_{n}=-m^{*} e^{4} / 2 \varepsilon_{0}^{2} \hbar^{2} n^{2}$ below the conduction band edge, where $m^{*}$ is the polaron mass of the electron. From the measured $1 s-n p$ transition energies we obtain the values of $m^{*}$ and the ionization energy $I_{1 s}$ of the $1 s$ state. It yields $m^{*} / m_{e o}=0.328$ and $I_{1 s}=84(89)$ $\mathrm{meV}$ for $\mathrm{Y}$ (In). Knowing the polaron mass we calculate the conduction band electron mass $m_{e}$, which gives $m_{e} / m_{e o}=0.237$, where $m_{e o}$ is the rest mass of the electron. If we calculate the ionization energy according to the bound polaron model [3] with this band mass, we get the value $78.1 \mathrm{meV}$, which is smaller than the experimentally observed energies for both $\mathrm{Y}$ and In donors. The increase of the ionization en- ergy can result from the attractive short-range potential of the donor and from the non-parabolicity of the conduction band. If the In atom substitutes the $\mathrm{Cd}$ atom in $\mathrm{CdF}_{2}$ crystal, then we are dealing with the isocoric impurity, for which the short-range potential should be negligibly small. We have therefore proposed a correction to the band shape taking on the conduction band dispersion relation in the form $E(k)=\hbar^{2} k^{2} / 2 m_{e}+\gamma k^{4}$. The value of the non-parabolicity parameter $\gamma$ has been chosen from the fit of the calculated and observed ionization energy for In donor. It leads to $\gamma=-11.44$ a.u. The chemical shift of donor levels (yttrium with respect to indium) is obtained by introducing the additional short-range potential $V_{\mathrm{SR}}(r)=C \delta(r)$, which reproduces the ionization energy of $\mathrm{Y}(84 \mathrm{meV})$ for $C=1.00$ a.u.

In order to study the properties of stable and bistable donors we have calculated the energy of $1 s$ state by variational means with the electronic wave function of the form $\phi_{1 s}(r) \sim \exp (-\beta r)$, where $\beta$ is equal to the inverse of the donor Bohr radius and plays a role of the localization parameter of the electron. The estimated values of $\beta$ are: 0.053 a.u. for $Y$ and 0.061 a.u. for In, i.e. the relative difference between both the values is $15 \%$ in comparison with $3 \%$ obtained in the hydrogen-like effective mass approximation for donors. The increase of the localization parameter for In is the reason of the considerable broadening and shift of the maximum of the infrared absorption band observed for this impurity.

We have as well calculated the absorption coefficient $\alpha$ as a function of the photon energy $h \nu$ in the region $0 \leq h \nu \leq 200 \mathrm{meV}$. The upper limit results from the range of applicability of the present approximations. For the yttrium donor we obtain a maximum of $\alpha(h \nu)$ for $h \nu=153 \mathrm{meV}$, which agrees with the experimental value of $160 \mathrm{meV}$ [1]. However, for the indium donor the maximum of the calculated $\alpha(h \nu)$ has not been reached in the above region, which means 
that the maximum lies at $h \nu \geq 200 \mathrm{meV}$ in comparison with the observed value of $235 \mathrm{meV}$.

The following picture can be helpful for the understanding of the large increase of the localization of $1 s$ state for the indium impurity. Figure 1 shows the dependence of the expectation value $E$ of the Hamiltonian of our system on the localization parameter $\beta$ for both impurities. Near the minimum, $E(\beta)$ varies more

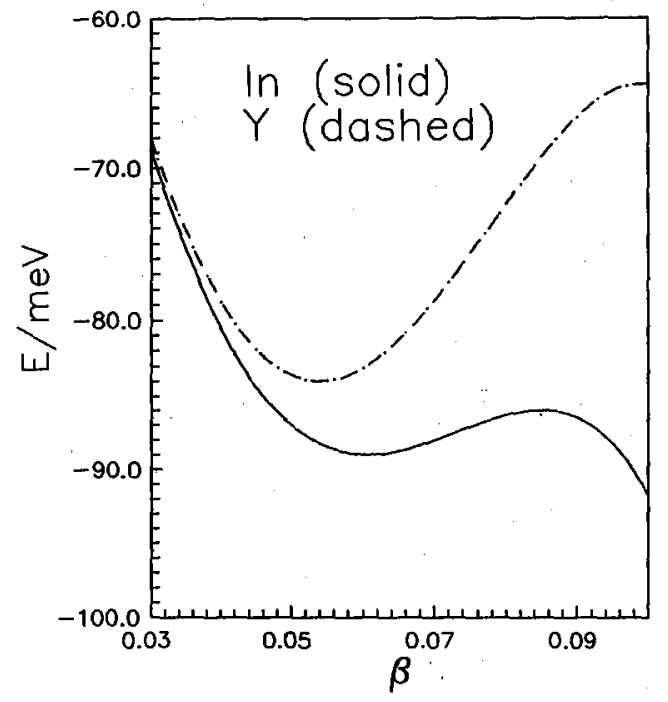

Fig. 1. Expectation value $E$ of the Hamiltonian of the donor as a function of the localization parameter $\beta$ for In (solid curve) and Y (dashed curve); the unit of $\beta$ is the inverse of the Bohr radius.

slowly for the indium than for the yttrium, which gives rise to the enlarged shift of the position of the minimum. Next, $E(\beta)$ increases with increasing $\beta$ up to a relatively low maximum and falls down below the value at the first local minimum. The global minimum will thus appear at the larger localization. This leads to the bistability of the indium donor, since - as it has been shown by one of the authors [2] - the phonon wave functions assure the orthogonality of the total wave functions for the weakly and strongly localized donor states. Therefore, the bistability of donors and the change of shape of the infrared absorption band have the common origin.

In this paper the results of the calculations of infrared absorption due to the two donors (In and $\mathrm{Y}$ ) in $\mathrm{CdF}_{2}$ crystals are presented. The values of input material parameters have been chosen from the discrete part of the donor spectrum. It is shown that the combined influence of the coupling with LO phonons, non-parabolicity of the conduction band and central-cell potential leads to the bistability of In donor as well as to the characteristic properties observed in the in-frared absorption in $\mathrm{CdF}_{2}$ crystals. 


\section{References}

[1] J.E. Dmochowski, J.M. Langer, W. Jantsch, in Shallow Impurities in Semiconductors 1988, ed. B. Monemar, Institute of Physics Conf. Series No. 95, IOP, Bristol and Philadelphia 1989, p. 325.

[2] S. Bednarek, Acta Phys. Pol. A77, 115 (1990).

[3] J. Adamowski, Phys. Rev. B 32, 2588 (1985). 